\title{
Socio-demographic differences in Colombian children's muscular fitness: Does scaling for differences in body size present a challenge to conventional thinking?
}

\author{
Alan M. Nevill' ${ }^{1}$ | Gavin Sandercock ${ }^{2}$ | Michael J. Duncan ${ }^{3}$ (D) | Ian Lahart ${ }^{1}$ | \\ Jorge Enrique Correa-Bautista ${ }^{4}$ ～Robinson Ramirez-Velez ${ }^{4}$ (C)
}

${ }^{1}$ Faculty of Education, Health and

Wellbeing, University of Wolverhampton,

Walsall Campus, Walsall,

United Kingdom

${ }^{2}$ School Sport, Rehabilitation and Exercise Science, University of Essex, Colchester, United Kingdom

${ }^{3}$ Faculty of Health and Life Sciences, Coventry University, Coventry,

United Kingdom

${ }^{4}$ Centro de Estudios para la Medición de la Actividad Física «CEMA». Escuela de Medicina y Ciencias de la Salud, Universidad del Rosario, Bogotá D.C, Colombia

Correspondence

Alan M. Nevill, University of Wolverhampton, Faculty of Education, Health and Wellbeing, Walsall Campus, Gorway Road, Walsall, WS1 3BD, United Kingdom.

Email: a.m.nevill@wlv.ac.uk

\begin{abstract}
Objectives: In low- to middle-income countries, children from less-deprived areas (from families of higher socio-economic status [SES]) have superior muscular fitness than those from low-SES groups. They are also taller and heavier, factors associated with muscular fitness. The purpose of this study was to identify any sociodemographic differences in Colombian children's muscular fitness and examine how these conclusions can be modified by scaling for differences in body size.

Methods: A total of 38,098 youths (46\% girls), ninth grade students (aged 14-15 years), participated in a study of cross-sectional design. We recorded SES and family incomes, stature, and mass. Standing broad jump and handgrip strength were used to assess muscular fitness. A multiplicative allometric model was adopted to adjust for body-size differences.

Results: Children from the mid- to high-SES groups jumped significantly higher than children from the lowest SES group, although no SES group difference in grip strength was observed. After adjusting for body size, children from higher SES and with higher family incomes had significantly lower handgrip strength, and their superior jump height performances remained but were greatly reduced. Only children from the highest SES now jumped significantly higher that the lowest SES group.

Conclusions: The superior jump performance and no difference in handgrip strength of Colombian children from higher SES may simply reflect their superior physiques. When body size is accounted for, these differences are reduced or even reversed, suggesting that children from higher SES groups should not be complacent regarding their apparent superior muscular fitness.
\end{abstract}

\section{1 | INTRODUCTION}

Musculoskeletal strength and power (muscular fitness) are recognized as important indicators of metabolic health status in youth (Artero et al., 2011; Grøntved et al., 2015; RamírezVélez et al., 2016; Steene-Johannessen, Anderssen, Kolle, \& Andersen, 2009). While associations between cardiorespiratory fitness and cardiovascular risk factors have been evaluated in youth from both high- and low-middle income countries (LMICs), studies examining interactions between muscular strength and health related risk factors have been conducted exclusively in high income countries (HICs), and largely in cohorts of European descent. There is a need to also assess these associations in LMICs, where there is a larger and more rapidly increasing burden of non-communicable disease (Cohen et al., 2014). The benefits of good muscular 
fitness are particularly evident in populations with excess adiposity or high body mass (Artero et al., 2011; Cohen et al., 2014; Cohen, López-Jaramillo, Fernández-Santos, CastroPiñero, \& Sandercock, 2017; Steene-Johannessen et al., 2009).

Exposure to social deprivation produces childhood health inequalities that may persist into adult life (Shishehbor, Gordon-Larsen, Kiefe, \& Litaker, 2008). We conceptualize socio-economic status (SES) as a combined measure of an individual's or family's economic and social position in relation to others, based on income, education, and occupation. Children from less-deprived areas (or from families of higher SES) have better aerobic and muscular fitness than those in low-SES groups (Jiménez-Pavón et al., 2010; Jin \& JonesSmith, 2015). Children in higher SES groups are also taller and have greater lean body mass than those of lower SES (Monyeki et al., 2005; Sandercock, Lobelo, \& CorreaBautista, 2017).

In developed nations, SES is positively associated with indices of childhood physical fitness (Jiménez-Pavón et al., 2010; Jin \& Jones-Smith, 2015). A more complex, curvilinear association appears to exist in LMICs (Petroski, Silva, \& De Lima, 2012; Petroski et al., 2012). Research has indicated that, in LMICs, family SES is inversely associated with physical fitness (Garber, Sajuria, and Lobelo, $20 \times 14$ ). SES disparity is considered the most fundamental cause of health disparities, and physical fitness is a predictor of adult health and disease (Leong et al., 2015). The PURE study of over 140,000 adults across low, middle, and high-income countries, reported that the lowest income countries had the lowest mean value for handgrip strength while highest income countries had the highest mean values (Leong et al., 2015).

The superior fitness of children from high-SES backgrounds has been attributed to differences in habitual physical activity between SES groups (Esmaeilzadeh, Kalantari, \& Nakhostin-Roohi, 2013; Jiménez-Pavón et al., 2010; Vandendriessche et al., 2012). Differences in muscular fitness could also be the product of SES-related differences in body dimensions (Monyeki et al., 2005; Sandercock et al., 2017).

Understanding how best to scale measures such as muscular fitness is important for researchers and policy makers in public health, education, and the exercise sciences (Nevill, Ramsbottom, \& Williams, 1992). Appropriate scaling helps ensure valid inferences can be made when investigating physiological differences in populations that also differ in terms of body size (Silva et al., 2016).

In LMICs, high SES children are taller and heavier than those with lower SES (Monyeki et al., 2005; Sandercock et al., 2017). Where SES differences in fitness are reported, there are often also differences in body size between the SES groups (Monyeki et al., 2005). Such body size differences may confound any analysis of how fitness may differ as a consequence of SES. Few studies adjust for the potential confounding effects of body mass (Cohen et al., 2014; Otero, Cohen, \& Herrera, 2017), none adjust for both stature and mass unless expressed as BMI in an attempt to adjust for adiposity. In LMICs, however, BMI is more strongly and positively associated with lean body mass than adiposity (Monyeki et al., 2005; Sandercock et al., 2017). It may, therefore, be important to account for both stature and body mass independently when attempting to understand how body size may influence muscular fitness in children from LMICs.

There is growing evidence that muscular fitness can aid prevention and treatment of metabolic disease independent of adiposity (Thivel, Ring-Dimitriou, Weghuber, Frelut, \& O'Malley, 2016). Allometric models provide valuable insight into the most appropriate body dimensions associated with children's physical performance and shape characteristics (Silva et al., 2016).

Allometric scaling may provide a more culturally specific method to understand the relative contribution of anthropometric and socio-demographic influences on the muscular fitness of children (Dos Santos et al., 2016). There is strong evidence that body size varies according to SES in Colombian children (Sandercock et al., 2017), but associations between SES and muscular fitness are less well-described (Cohen et al., 2014; Zhang \& Martinez-Donate, 2017). To date, there are no data on the scaling of muscular fitness in Colombian children. This study sought to address this issue by identifying any socio-demographic differences in Colombian children's muscular fitness and examining how these conclusions might be influenced by appropriately scaling for differences in body size.

\section{2 | MATERIALS AND METHODS}

\section{1 | Study design and participants}

The sample consisted of children drawn from the combined "Curriculum $40 \times 40$ " and "Prueba Ser" surveys administered by Bogota's District Secretary of Education in November, 2015. These were cross-sectional surveys of ninth grade students (aged 14 to 15 years) recruited from public and private schools in all 20 "localidades" (municipalities) within the District Capital of Bogota (Cundinamarca Department, Andean Region of Colombia). The Study was approved by the Review Committee for Research in Human Subjects at the University of Rosario (Code N ${ }^{\circ}$ CEI-ABN026-000262). The nature and purpose of the study were given to potential participants and their parents or guardians explaining that data would be available to the Colombian Health Authorities in accordance with the Law of Data Protection (Resolution 8430/93). Further details regarding the sample and study design can be obtained here: http://www.educacionbogota.edu.co/ 
archivos/Temas\%20estrategicos/Documentos/Resultados_PruebasSER-Bienestar_Fisico_Ciudadania_y_Convivencia.pdf .

\subsection{Anthropometric variables}

Data were collected at the same time in the morning (between 7:00 a.m. and 10:00 a.m.) following an overnight fast in accordance with the ISAK (International Society for the Advancement of Kinanthropometry) guidelines (Stewart, Marfell-Jones, \& Olds, 2011). Mass was measured to the nearest $0.10 \mathrm{~kg}$ with the participant lightly dressed using a portable electronic weight scale (Tanita ${ }^{\circledR}$ BC544, Tokyo, Japan) with a low technical error of measurement (TEM = 0.510). Stature was measured to the nearest $0.1 \mathrm{~cm}$ in bare or stocking feet with the adolescent standing upright against a portable stadiometer (Seca ${ }^{\circledR}$ 274, Hamburg, Germany; $\mathrm{TEM}=0.019$ ).

\subsection{Social economic status and family income}

SES was determined using the System of Identifying Potential Beneficiaries of Social Programs (SISBEN, 1-6 on a scale defined by the Colombian authorities) (The World Bank, 2005). SISBEN is a composite score based on sociodemographic characteristics (family composition, employment status, level of educational); living conditions (dwelling type, construction materials); and access to public utilities (sewerage, electricity, potable water, refuse collection). Note that the term "dwelling" refers to where the children lived rather than where they went to school, and the term "family composition" refers to a person or group of persons that live in a house or part of it and share food or food budget. Level of education is classified based on the median level of education attained by residents in each given geographical area. Households are ranked or split into 6 strata with SISBEN 1very low, 2-low, and 3-medium-low the most vulnerable and targeted in social programs. SISBEN level 4-medium, 5medium high, and 6-high strata are considered the least vulnerable sectors of society. SISBEN 4-6 are regarded as wealthy, and for the purposes of the present study we conceptualized SES as 4 categories "Very Low," "Low," and "Medium," corresponding to SISBEN categories 1-3, and "High" corresponding to SISBEN categories 4-6. Parental income data were used to create the variable "Family Income" according to the classification used by the District Secretary of Education and SISBEN at the time of enrolling the child in school in January, 2014. This variable was grouped into three categories as 1 (low, $\leq \$ 205$ USD per month), 2 (middle, $>\$ 205<\$ 410$ USD per month), and 3 (high $>\$ 410$ USD per month). "Family income" reflected the average monthly income of all members of the household (i.e., both parents). The average monthly income for Colombia is $\$ 380$ USD.

\section{4 $\mid$ Muscular fitness measurement}

Musculoskeletal fitness was assessed using two tests. The standing broad jump (Jump) was used assess lower body muscular fitness. Participants were instructed to jump as far as possible using a two footed take-off and landing technique. They were encouraged to flex then extend their knees, ankles, and hips and to swing their arms to maximize performance. Test performance was assessed by measuring the distance between participants' toes at take off to the heel at landing. Participants were required to execute two jumps using the correct technique with performance recorded as the greatest distance achieved in either jump. The reproducibility of our data reached $R=0.78$.

Handgrip strength was assessed as an indicator of upperbody muscular fitness using an adjustable analog handgrip dynamometer (Takei Scientific Instruments Co., Ltd, Niigata, Japan). Researchers provided standardized verbal instructions describing the correct execution of the test before providing a visual demonstration of the technique. Dynamometers were adjusted to account for differences in hand size. Handgrip strength was measured with the subject in a standing position with the shoulder adducted and neutrally rotated and arms parallel but not in contact with the body. The participants were asked to squeeze the handle for a maximum of 3-5 seconds. No verbal encouragement was given during the test. Participants performed two trials with each hand. The values used in the present study represent the highest value obtained with either hand. This procedure was employed as asymmetry in hand grip strength as a function of hand dominance is rare in children of the ages taking part in the present study (Butterfield, Lehnhard, Loovis, Coladarci, \& Saucier, 2009). The reproducibility of our data was $R=0.96$.

\section{5 | Statistical methods}

An appropriate method of analyzing hierarchical data (children nested within schools) is to adopt a multilevel modeling approach using the Statistical Software MLwiN version 2.36. Multilevel modeling is an extension of ordinary multiple regression where the data have a hierarchical or clustered structure. A hierarchy consists of units or measurements grouped at different levels. In the current study, the multilevel regression analyzes were performed to identify those factors (differences in SES, family income, rural versus urban, etc.) associated with the development of hand-grip strength and jump performance, respectively, both before and after adjusting for differences in body size (stature and mass). The two levels of hierarchical or nested observational units used 
TABLE 1 Characteristics of (a) boys and (b) girls by socio-economic status, family income, and dwelling

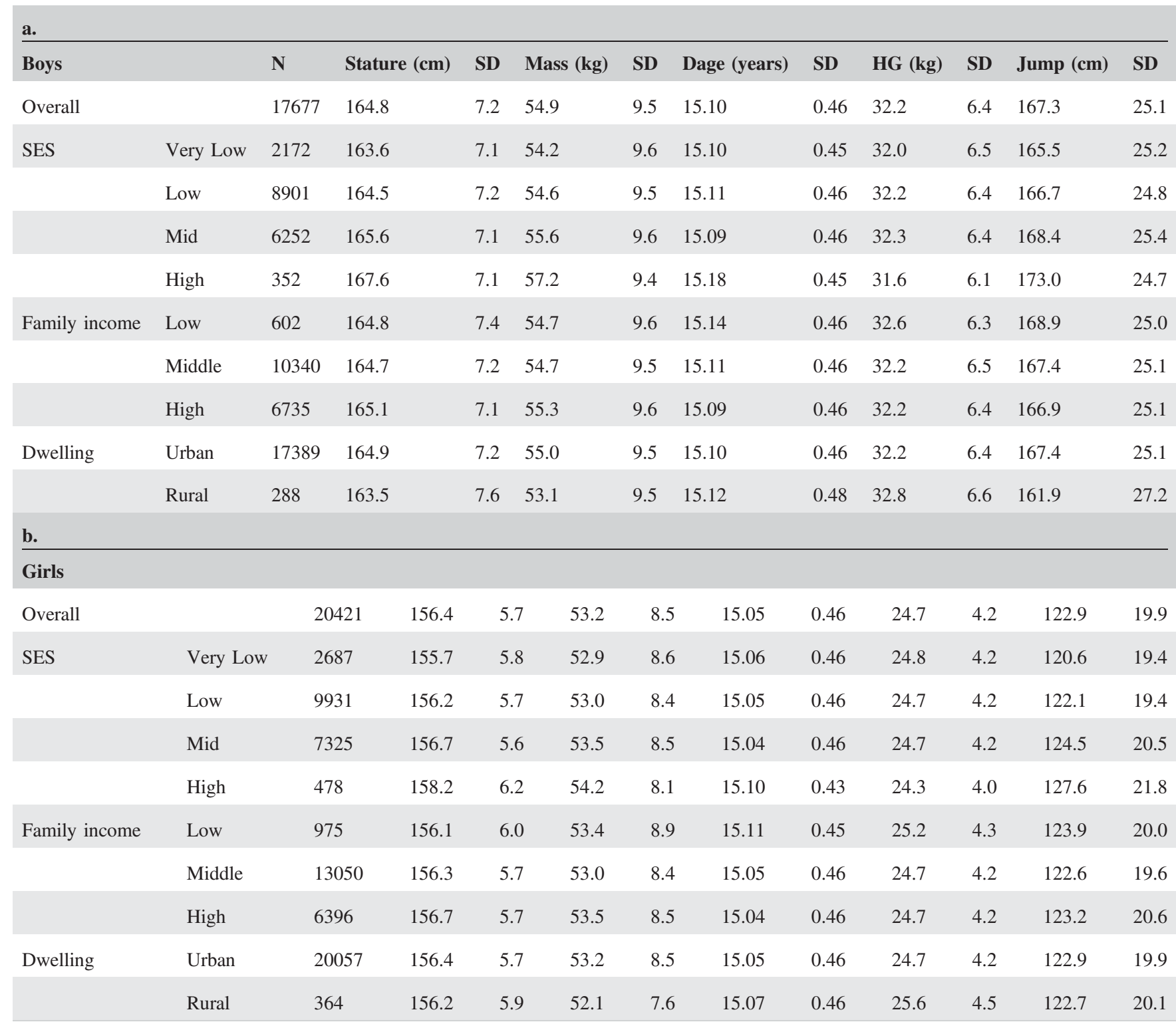

Key: SES = socio-economic status; DAage $=$ decimal age; HG = handgrip strength; Jump $=$ Standing broad jump height.

in the analyzes were, (i) the children at level 1 (within schools), and (ii) between the schools at level 2 .

Human strength and work capacity increases proportionally with body size (Bustamante Valdivia, Maia, \& Nevill, 2015; Nevill et al., 1998). Adapting a multiplicative, allometric model structure that has been used to describe a variety of human performance indices, such as hand grip strength (Nevill \& Holder, 2000), strength and aerobic power (Nevill et al., 1998), and physical performance (Bustamante Valdivia et al., 2015), the proposed model for the grip strength and jump performances of the Colombian ninth grade children (aged 14 to 15 years) measurements $(\mathrm{Y})$ is given as follows:

$$
Y=\text { stature }^{\mathrm{k}}{ }_{1} \cdot \operatorname{mass}_{2}^{\mathrm{k}} \cdot \exp \left(b_{0}+b_{1} \cdot \operatorname{age}\right) .
$$

The model (Equation 1) can be linearized with a log transformation using natural $\operatorname{logs}(\mathrm{Ln})$. A linear regression analysis on $\operatorname{Ln}(Y)$, can then be used to estimate the unknown parameters of the log transformed model (Equation 2).

$$
\operatorname{Ln}(Y)=k_{1} \cdot \operatorname{Ln}(\text { stature })+k_{2} \cdot \operatorname{Ln}(\text { mass })+b_{0}+b_{1} \cdot \text { age }
$$

Further categorical or group differences within the population (e.g., differences in SES, family income, rural versus urban, etc.) can easily be explored by allowing some of the parameters in the log transformed model (Equation 2) to vary for each group (by introducing fixed indicator factors).

The proportional model (Equation 1) also assumes that the residual errors of $\mathrm{Y}$ are heteroscedastic (proportional), that is, the error variance will increase with larger measurement 
TABLE 2 The multilevel regression analysis of log-transformed (using natural logs Ln) hand-grip strength of 9th grade Colombian boys and girls, (i) after adjusting for only decimal age (Dage) and (ii) after adjusting for body size (mass and stature) and decimal age

\begin{tabular}{|c|c|c|c|c|c|}
\hline \multirow[b]{2}{*}{ Fixed explanatory factors } & \multicolumn{2}{|l|}{ Model (i) } & \multirow[b]{2}{*}{ Fixed explanatory factors } & \multicolumn{2}{|l|}{ Model (ii) } \\
\hline & Estimate & SE & & Estimate & SE \\
\hline Constant $\left(b_{0}\right)$ & 2.3780 & 0.0316 & Constant $\left(b_{0}\right)$ & 0.6214 & 0.0313 \\
\hline FI Mid $\left(\Delta b_{0}\right)$ & -0.0103 & 0.0048 & FI Mid $\left(\Delta b_{0}\right)$ & -0.0078 & 0.0040 \\
\hline FI High $\left(\Delta b_{0}\right)$ & -0.0068 & 0.0049 & FI High $\left(\Delta b_{0}\right)$ & -0.0090 & 0.0041 \\
\hline Rural $\left(\Delta b_{0}\right)$ & 0.0250 & 0.0133 & Rural $\left(\Delta b_{0}\right)$ & 0.0386 & 0.0133 \\
\hline SES Low $\left(\Delta b_{0}\right)$ & 0.0049 & 0.0061 & SES Low $\left(\Delta b_{0}\right)$ & -0.0009 & 0.0061 \\
\hline $\operatorname{SES} \operatorname{Mid}\left(\Delta b_{0}\right)$ & 0.0058 & 0.0062 & SES Mid $\left(\Delta b_{0}\right)$ & -0.0135 & 0.0063 \\
\hline SES High $\left(\Delta b_{0}\right)$ & -0.0086 & 0.0114 & SES High $\left(\Delta b_{0}\right)$ & -0.0439 & 0.0112 \\
\hline Girls $\left(\Delta b_{0}\right)$ & -0.2536 & 0.0019 & Girls $\left(\Delta b_{0}\right)$ & -0.1747 & 0.0019 \\
\hline \multirow[t]{3}{*}{ Dage } & 0.0713 & 0.0020 & Dage & 0.0420 & 0.0017 \\
\hline & & & Ln (Mass) & 0.3869 & 0.0058 \\
\hline & & & Ln (Stature) & 1.3310 & 0.0231 \\
\hline Variance of random factors & Constant (a) & SE & & Constant (a) & SE \\
\hline Level 2 (Schools) & 0.0012 & 0.0001 & Level 2 (Schools) & 0.0014 & 0.0001 \\
\hline Level 1 (Individuals) & 0.0324 & 0.0002 & Level 1 (Individuals) & 0.0225 & 0.0002 \\
\hline
\end{tabular}

Values are means \pm SE. Hand grip strength is recorded in $\mathrm{kg}$ and entered as [ $\mathrm{Ln}(\mathrm{kg})]$. Age was measures as decimal age (Dage) in years. Boys from the very lowest SES, with the lowest family income (FI) and from an urban background were used as the constant baseline measure in equation $2_{0}$, and other groups were compared with it, indicated by $\left(\Delta b_{0}\right)$.

means and conversely decrease with smaller means. Thus, by fitting the parameters using log-linear regression (Equation 2), it is assumed that the residual error variance of the log transformed handgrip strength and jump performance measurements remains constant throughout the range of observations.

\section{3 | RESULTS}

In our sample of 38,098 youths (46\% girls and $54 \%$ boys), girls were $5 \%$ shorter, weighed $3 \%$ less, achieved handgrip strength scores $23 \%$ lower, and jumped 26\% lower compared to boys (see Table 1a,b). Importantly, boys and girls from the higher SES groups were both taller and heavier than children from the lower SES groups.

\section{1 | Handgrip results}

Compared to our baseline group (boys from the very lowest SES, with the lowest family income, and from an urban background), there were little or no significant differences in handgrip strength (log transformed) across all other groups other than girls having approximately 25\% lower handgrip $\left(\Delta b_{0}=-0.25 \mathrm{SE}=0.0019\right)$ after adjusting for age. Hand grip strength increased significantly at approximately $7 \%$ per year $\left(b_{1}=0.071, \mathrm{SE}=.002\right)$ (Table 2, Model i).

However, after adjusting for both body size (mass and stature) and decimal age, we found that children from higher SES and with higher family incomes had significantly lower handgrip strength (Table 2, Model ii). Also, children living in rural locations had significantly higher handgrip strength $4 \%\left(\Delta b_{0}=0.0386, \mathrm{SE}=.0133\right)$ than children living in urban locations. After adjusting for both body size and age, girls had approximately $17 \%$ lower handgrip strength and the children's handgrip strength increased at a lower, but still significant rate of approximately $4 \%$ per year (body size explaining both these (sex and age) reductions in hand grip performances observed in model I earlier). This indicates that handgrip strength increases at a rate of $4 \%$ per year having already controlled for body size.

Note that the contributions of log-transformed mass and stature were both positive and highly significant (see Table 2, Model ii).

\section{2 | Jump results}

Compared to our baseline group (boys from the very lowest SES, with the lowest family income, and from an urban 
TA B LE 3 The multilevel regression analysis of log-transformed (using natural logs Ln) jump height of 9th grade Colombian boys and girls, (i) after adjusting for only decimal age and (ii) after adjusting for body size (mass and stature) and decimal age (Dage)

\begin{tabular}{|c|c|c|c|c|c|}
\hline \multirow[b]{2}{*}{ Fixed explanatory factors } & \multicolumn{2}{|l|}{ Model (i) } & \multirow[b]{2}{*}{ Fixed explanatory variables } & \multicolumn{2}{|l|}{ Model (ii) } \\
\hline & Estimate & SE & & Estimate & SE \\
\hline Constant $\left(b_{0}\right)$ & 4.7200 & 0.0270 & Constant $\left(b_{0}\right)$ & 4.9860 & 0.0308 \\
\hline FI Mid $\left(\Delta b_{0}\right)$ & 0.0003 & 0.0040 & FI Mid $\left(\Delta b_{0}\right)$ & -0.0009 & 0.0039 \\
\hline FI High $\left(\Delta b_{0}\right)$ & 0.0025 & 0.0041 & FI High $\left(\Delta b_{0}\right)$ & 0.0014 & 0.0040 \\
\hline Rural $\left(\Delta b_{0}\right)$ & -0.0153 & 0.0174 & Rural $\left(\Delta b_{0}\right)$ & -0.0166 & 0.0172 \\
\hline SES Low $\left(\Delta b_{0}\right)$ & 0.0103 & 0.0082 & SES Low $\left(\Delta b_{0}\right)$ & 0.0058 & 0.0081 \\
\hline SES Mid $\left(\Delta b_{0}\right)$ & 0.0202 & 0.0084 & $\operatorname{SES} \operatorname{Mid}\left(\Delta b_{0}\right)$ & 0.0131 & 0.0083 \\
\hline SES High $\left(\Delta b_{0}\right)$ & 0.0528 & 0.0142 & SES High $\left(\Delta b_{0}\right)$ & 0.0380 & 0.0141 \\
\hline Girls $\left(\Delta b_{0}\right)$ & -0.3077 & 0.0016 & Girls $\left(\Delta b_{0}\right)$ & -0.2586 & 0.0019 \\
\hline \multirow[t]{3}{*}{ Dage } & 0.0246 & 0.0017 & Dage & 0.0216 & 0.0017 \\
\hline & & & Ln (Mass) & -0.1843 & 0.0056 \\
\hline & & & Ln (Stature) & 1.0460 & 0.0224 \\
\hline Variance of Random variable & Constant (a) & SE & & Constant (a) & SE \\
\hline Level 2 (Schools) & 0.0028 & 0.0002 & Level 2 (Schools) & 0.0028 & 0.0002 \\
\hline Level 1 (Individuals) & 0.0224 & 0.0002 & Level 1 (Individuals) & 0.0211 & 0.0002 \\
\hline
\end{tabular}

Values are means \pm SE. Jump height was recorded as $\mathrm{cm}$ and entered as $[\mathrm{Ln}(\mathrm{cm})]$. Age was measures as decimal age (Dage) in years. Boys from the very lowest SES, with the lowest family income and from an urban background were used as the constant baseline measure in equation $2_{0}$ ), and other groups were compared with it, indicated by $\left(\Delta b_{0}\right)$.

background), children from the mid- to high-SES groups jumped significantly higher than children from lower SES groups. Girls also jumped approximately $31 \%$ lower $\left(\Delta b_{0}=-0.31 \mathrm{SE}=0.0016\right)$ than boys after adjusting for age alone. No difference in mean jump heights were observed between family-income groups nor urban vs rural locations. Jump height also increased significantly at approximately $2.5 \%$ per year $\left(b_{1}=0.0246, \mathrm{SE}=.0017\right)$ (Table 3 , Model i).

After adjusting for both body size (mass and stature) as well as decimal age, we found that the differences observed in model ii remained but were greatly reduced. Only children from the highest SES group jumped significantly higher that the baseline SES group. As observed in model $i$, no difference in mean jump heights were observed between familyincome groups nor urban $v s$ rural locations. After adjusting for body size, girls jumped approximately $26 \%$ lower $\left(\Delta b_{0}=-0.259 \mathrm{SE}=0.0019\right)$ than boys having also adjusted for age. Jump height increased significantly at a slightly reduced rate of approximately $2.2 \%$ per year $\left(b_{1}=0.0216\right.$, $\mathrm{SE}=.0017)$ (Table 3, Model ii). Once again, body size can explain both these (sex and age) reductions in jump height performance observed in Table 3, Model i described earlier).
Note that the contributions of log-transformed mass and stature now had opposite signs and were both highly significant (see Table 3, Model ii).

\section{4 | DISCUSSION}

This study presents novel data, using an allometric scaling model, to better understand differences in muscular fitness in Colombian children from different socio-demographic backgrounds. This is the first study to present such data in Colombian children and as such extends the body of knowledge relating to pediatric health and fitness related to SES. The current study suggests that, if body size is not accounted for, there are no differences in handgrip strength between SES or family income groups nor between children from urban or rural areas. Stature and body mass were, however, greater in urban dwelling children and those from higher SES groups. Handgrip strength increased as a function both of stature and body mass. To obtain a more accurate interpretation of socio-demographic differences, body size must be accounted for. After adjusting for differences in body size, 
we found urban children, those with high SES, and higher family income had lower handgrip strength. It would appear that children from higher SES groups underperform at the handgrip strength test proportional to their (larger) body dimensions. This finding is at odds with studies in HICs (Jiménez-Pavón et al., 2010; Jin \& Jones-Smith, 2015) and LMICs (Petroski et al., 2012; Petroski, da Silva, \& Rodrigues, 2011), which report better handgrip in middle or high SES children.

The body mass and stature exponents reported (Table 2) are $0.38(\mathrm{SE}=0.006)$ and $1.33(\mathrm{SE}=0.02)$, respectively, both a little greater than a linear $\left(L=M^{0.33}\right)$ dimension of body size (see Astrand \& Rodahl, 1986). Taken together the product approximates $L^{2}$, which can be interpreted as the cross sectional or surface area of the children's body size dimension. No study of SES has adjusted children's handgrip both for body mass and stature; despite clear evidence that both measures vary according to SES.

Our results illustrate the importance of appropriate scaling using the allometric approach to avoid making erroneous conclusions as to the association between fitness and sociodemographic factors that are actually explained by differences in body dimensions.

Regarding handgrip scores, our results agree with another recent study (Otero et al., 2017) which found Colombian youth with poor handgrip strength were more likely to be from higher SES groups. The authors reported lower BMI (z-scores) in children with the poorest handgrip strength but found no meaningful between-group differences in adiposity (skinfold thickness) (Otero et al., 2017).

The variation in children's stature associated with SES results in difficulty interpreting the between-group differences in BMI (Monyeki et al., 2005; Sandercock et al., 2017; Silva et al., 2016). The model used to predict handgrip included positive exponents for both body mass and stature, as shown previously in studies of South American (Silva et al., 2016), European (Nevill, Tsiotra, Tsimeas, \& Koutedakis, 2009; Tambalis, Panagiotakos, \& Sidossis, 2011) and African (Dos Santos et al., 2016) youth. The importance of appropriate scaling when comparing cohorts of different body size is also highlighted in a recent cross-cultural comparison showing higher absolute handgrip values in youth from Portugal compared with Mozambique (Dos Santos et al., 2016). However, once concurrent differences in body size were accounted for, the direction of handgrip differences was reversed.

Greater stature is advantageous to horizontal jumping performance (Dos Santos et al., 2016), but, in contrast to handgrip strength, in the current study we found the exponent for body mass was negative. This resulted in the performance difference for jumping between high and low SES children being reduced once data were adjusted for body size.
Participants in the highest SES group jumped 5.3\% further than those from the lowest group. Adjusting for body size attenuated the SES-related differences in jump performance, but high SES children still jumped 3.8\% further than the lowest group. Positive associations of SES with muscular fitness have been reported in HICs and LMIC youth.

Regardless of whether we adjusted for body size, there were no significant differences in jump performance by family income or rural versus urban dwelling. These findings conflict with studies in LMICs that report better muscular fitness in urban rather than rural dwelling youth (Andrade et al., 2014; Garber, Sajuria, \& Lobelo, 2014; Pena Reyes et al., 2003; Tambalis et al., 2011; Ujevic, Sporis, \& Milanovic, 2013), but only one study of Greek youth has scaled for body size (Tambalis et al., 2011).

However, probably one of the most illuminating findings obtained from jump height [model (ii)] was identified with the body mass and stature exponents given in Table 3 . These were $-0.18(\mathrm{SE}=0.006)$ and $1.05(\mathrm{SE}=0.02)$, respectively. Note that they have opposite signs. After taking ant-logs, these opposite signs indicate that the association between jump height and body size is a "height-to-weight" ratio, given by stature/(mass $)^{0.18}$. This empirically derived ratio is not dissimilar to the reciprocal Ponderal index, RPI $=$ stature/(mass $)^{0.333}$, well known for being a strong indicator of athletic performance (Watts, Coleman, \& Nevill, 2012).

Scaling is an important consideration when working with physical fitness or performance data in pediatric populations as it can reveal differences between groups with different body dimensions (Dos Santos et al., 2016) that otherwise would not have been identified. Body mass and stature are both important determinants of handgrip strength and horizontal jump performance; two of the most frequently used field based measures of muscular fitness. However, research fails to adequately consider the impact of stature and body mass on performance of these tests. While prior research has adjusted (as covariates) for variables such as BMI or skinfolds (Jiménez-Pavón et al., 2010), stature is an important predictor of handgrip strength and many studies only correct for body mass when examining handgrip data. Scaling handgrip for body mass at a 1:1 ratio results in a negative association between mass and the resultant variable and may penalize heavier individuals unfairly. As a consequence it is important for scientists, epidemiologists and public health practitioners to consider both body mass and stature when examining differences in muscular fitness between groups.

The present study illustrates this point elegantly in the context of SES differences in muscular fitness in Colombian children. As high SES children have a physical advantage over their low SES peers, they should theoretically perform better than low SES children. When adjusting for body size, high SES children demonstrate considerably lower muscular strength; while variations in jump performance persisted, 
differences between high and low SES groups became less pronounced. We acknowledge that these results are specific to Colombian children.

The results of the current study provide evidence supporting the utility of scaling as a means to better understand the impact of SES on children's muscular fitness in LMICs. Our findings suggest the superior muscular fitness of high SES children is attributable to superior physique demonstrated in the high SES group rather than being a result of SES per se.

\section{AUTHOR CONTRIBUTIONS}

AMN, MJD, IL, GS analyzed the data and drafted the manuscript. AMN, GS, JEC-R, RR-V designed the study, directed implementation and data collection. AMN, MJD, IL, JEC-R, RR-V, and GS edited the manuscript for intellectual content and provided critical comments on the manuscript.

\section{CONFLICT OF INTERESTS}

None.

\section{ORCID}

Michael J. Duncan (D) http://orcid.org/0000-0002-2016-6580

Robinson Ramirez-Velez (1) http://orcid.org/0000-0003-30756960

\section{REFERENCES}

Andrade, S., Lachat, C., Ochoa-Aviles, A., Verstraeten, R., Huybregts, L., Roberfroid, D., ... Kolsteren, P. (2014). A schoolbased intervention improves physical fitness in Ecuadorian adolescents: A cluster-randomized controlled trial. International Journal of Behavioral Nutrition and Physical Activity, 11(1), 153.

Artero, E. G., Ruiz, J. R., Ortega, F. B., España-Romero, V., Vicente-Rodríguez, G., Molnar, D., ... Gutiérrez, A. (2011). Muscular and cardiorespiratory fitness are independently associated with metabolic risk in adolescents: The HELENA study. Pediatric Diabetes, 12(8), 704-712.

Astrand, P.-O., \& Rodahl, K. (1986). Textbook of work physiology. 3rd ed. New York: McGraw-Hill.

Bustamante Valdivia, A., Maia, J., \& Nevill, A. (2015). Identifying the ideal body size and shape characteristics associated with children's physical performance tests in Peru. Scandinavian Journal of Medicine \& Science in Sports, 25(2), e155-e165.

Butterfield, S. A., Lehnhard, R. A., Loovis, E. M., Coladarci, T., \& Saucier, D. (2009). Grip strength performance by 5- to 19-year olds. Perceptual and Motor Skills, 109(2), 362-370.

Cohen, D. D., Gómez-Arbeláez, D., Camacho, P. A., Pinzon, S., Hormiga, C., Trejos-Suarez, J., ... Lopez-Jaramillo, P. (2014). Low muscle strength is associated with metabolic risk factors in Colombian children: The ACFIES Study. Plos One, 9(4), e93150.
Cohen, D. D., López-Jaramillo, P., Fernández-Santos, J. R., CastroPiñero, J., \& Sandercock, G. R. H. (2017). Muscle strength is associated with lower diastolic blood pressure in schoolchildren. Preventive Medicine, 95, 1-6.

dos Santos, F. K., Nevill, A., Gomes, T. N. Q. F., Chaves, R., Daca, T., Madeira, A., ... Maia, J. A. R. (2016). Differences in motor performance between children and adolescents in Mozambique and Portugal: Impact of allometric scaling. Annals of Human Biology, 43(3), 191-200.

Esmaeilzadeh, S., Kalantari, H., \& Nakhostin-Roohi, B. (2013). Cardiorespiratory fitness, activity level, health-related anthropometric variables, sedentary behaviour and socioeconomic status in a sample of Iranian 7-11 year old boys. Biology of Sport, 30(1), 67-71.

Garber, M. D., Sajuria, M. F., \& Lobelo, F. (2014). Geographical variation in health-related physical fitness and body composition among chilean 8th graders: A Nationally representative crosssectional study. Plos One, 9(9), e108053.

Grøntved, A., Ried-Larsen, M., Møller, N. C., Kristensen, P. L., Froberg, K., Brage, S., \& Andersen, L. B. (2015). Muscle strength in youth and cardiovascular risk in young adulthood (the European Youth Heart Study). British Journal of Sports Medicine, 49(2), 90-94.

Jiménez-Pavón, D., Ortega, F. B., Ruiz, J. R., Chillón, P., Castillo, R., Artero, E. G., ... Gonzalez-Gross, M. (2010). Influence of socioeconomic factors on fitness and fatness in Spanish adolescents: The AVENA study. International Journal of Pediatric Obesity, 5(6), 467-473.

Jin, Y., \& Jones-Smith, J. C. (2015). Associations between family income and children's physical fitness and obesity in California, 2010-2012. Preventing Chronic Disease, 12, E17.

Leong, D. P., Teo, K. K., Rangarajan, S., Lopez-Jaramillo, P., Avezum, A., Orlandini, A., ... Yusuf, S. (2015). Prognostic value of grip strength: Findings from the Prospective Urban Rural Epidemiology (PURE) study. The Lancet, 386(9990), 266-273.

Monyeki, M. A., Koppes, L. L. J., Kemper, H. C. G., Monyeki, K. D., Toriola, A. L., Pienaar, A. E., \& Twisk, J. W. R. (2005). Body composition and physical fitness of undernourished South African rural primary school children. European Journal of Clinical Nutrition, 59(7), 877-883.

Nevill, A. M., Holder, R. L., Baxter-Jones, A., Round, J. M., \& Jones, D. A. (1998). Modeling developmental changes in strength and aerobic power in children. Journal of Applied Physiology, 84 (3), 963-970.

Nevill, A. M., Ramsbottom, R., \& Williams, C. (1992). Scaling physiological measurements for individuals of different body size. European Journal of Applied Physiology and Occupational Physiology, 65(2), 110-117.

Nevill, A. M., \& Holder, R. L. (2000). Modelling handgrip strength in the presence of confounding variables: Results from the Allied Dunbar National Fitness Survey. Ergonomics, 43(10), 1547-1558.

Nevill, A., Tsiotra, G., Tsimeas, P., \& Koutedakis, Y. (2009). Allometric associations between body size, shape, and physical performance of Greek children. Pediatric Exercise Science, 21(2), 220-232.

Otero, J., Cohen, D. D., \& Herrera, V. M. (2017). Sociodemographic factors related to handgrip strength in children and adolescents in 
a middle income country: The SALUS study. American Journal of Human Biology, 29, e-pub ahead of print.

Pena Reyes, M. E., Tan, S. K., \& Malina, R. M. (2003). Urban-rural contrasts in the physical fitness of school children in Oaxaca, Mexico. American Journal of Human Biology, 15(6), 800-813.

Petroski, E., da Silva, A. F., \& Rodrigues, A. B. (2011). [Healthrelated physical fitness in Brazilian adolescents from areas having a medium/low Human Development Index]. Review Salud Publica (Bogota), 13, 219-228.

Petroski, E., Silva, D., \& De Lima, E. S. (2012). Health-related physical fitness and associated sociodemographic factors in adolescents from a Brazilian state capital. Human Movement, 13, 139-146.

Ramírez-Vélez, R., Anzola, A., Martinez-Torres, J., Vivas, A., Tordecilla-Sanders, A., Prieto-Benavides, D., ... Garcia-Hermoso, A. (2016). Metabolic syndrome and associated factors in a population-based sample of schoolchildren in Colombia: The FUPRECOL Study. Metabolic Syndrome and Related Disorders, 14(9), 455-462.

Sandercock, G., Lobelo, F., \& Correa-Bautista, J. (2017). The relationship between socioeconomic status, family income, and measures of muscular and cardiorespiratory fitness in Colombian schoolchildren. Journal of Pediatrics, 185, 81-87.

Shishehbor, M. H., Gordon-Larsen, P., Kiefe, C. I., \& Litaker, D. (2008). Association of neighborhood socioeconomic status with physical fitness in healthy young adults: The Coronary Artery Risk Development in Young Adults (CARDIA) study. American Heart Journal, 155(4), 699-705.

Silva, S., Bustamante, A., Nevill, A., Katzmarzyk, P. T., Freitas, D., Prista, A., \& Maia, J. (2016). An allometric modelling approach to identify the optimal body shape associated with, and differences between Brazilian and Peruvian youth motor performance. PLoS One, 11(3), e0149493.

Steene-Johannessen, J. S., Anderssen, A., Kolle, E., \& Andersen, L. B. (2009). Low muscle fitness is associated with metabolic risk in youth. Medicine and Science in Sports and Exercise, 41(7), 1361-1367.

Stewart, A., Marfell-Jones, M., \& Olds, T. (2011). International Standards for Anthropometric Assessment. Lower Hutt, New
Zealand: International Society for the Advancement of Kinanthropometry.

Tambalis, K. D., Panagiotakos, D. B., \& Sidossis, L. S. (2011). Greek children living in rural areas are heavier but fitter compared to their urban counterparts: A comparative, time-series (19972008) analysis. The Journal of Rural Health, 27(3), 270-277.

The World Bank (2005). Targeting Social Spending To The Poor With Proxy-Means Testing: Colombia's SISBEN System. Washington DC: The World Bank.

Thivel, D., Ring-Dimitriou, S., Weghuber, D., Frelut, M.-L., \& O'Malley, G. (2016). Muscle strength and fitness in pediatric obesity: A systematic review from the European childhood obesity group. Obesity Facts, 9(1), 52-63.

Ujevic, T., Sporis, G., \& Milanovic, Z. (2013). Differences between health-related physical fitness profiles of Croatian children in urban and rural areas. Collegium Antropologicum, 37, 75-80.

Vandendriessche, J. B., Vandorpe, B. F. R., Vaeyens, R., Malina, R. M., Lefevre, J., Lenoir, M., \& Philippaerts, R. M. (2012). Variation in sport participation, fitness and motor coordination with socioeconomic status among Flemish children. Pediatric Exercise Science, 24(1), 113-128.

Watts, A., Coleman, I., \& Nevill, A. (2012). The changing shape characteristics associated with success in world-class sprinters. Journal of Sports Science, 30(11), 1085-1095.

Zhang, X., \& Martinez-Donate, A. P. (2017). Socioeconomic status and youth physical fitness: Evidence from an upper-middle income country. Journal of Pediatrics, 185, 14-16. 